\title{
Fiber Dynamical Analysis of Twist Draft Spinning
}

\section{TAKEMURA Hidetaka*, NAKAZAWA Masaru** and KAWAMURA Takashi**}

\author{
*Dep. of Control Engineering, Nagano Prefectural Institute of Technology, 813-8 Shimonogo, Ueda, Nagano, 386-1211 Japan \\ **Faculty of Textile Science and Technology, Shinshu University, 3-15-1 Tokida, Ueda, Nagano, 386-8567 Japan
}

Received; Accepted for Publication

\begin{abstract}
This paper presents an analysis of the fiber dynamics in the twist draft spinning, and an application of the derived theory from the analysis to TCTDS (Tension Control type Twist Draft Spinning), a kind of the twist draft spinning that is being developed by the authors.

Through the analysis, we specially have regard for linear density and twist rate of the twist draft spinning yarn those are deeply concerned with the quality and structure of the yarn. And we found that the twist multiplier of the twist draft spinning yarn spun in steady state of the spinning process is formulated by a function of properties of raw materials, e.g., fiber length, fiber diameter, and the coefficient of inter-fiber friction.

The theoretical result shows good agreement with the practical data of yarn properties given by the related experiments, and it proves to be a valuable tool in designing of yarns spun with TCTDS.
\end{abstract}

Keywords: twist draft spinning, fiber dynamical analysis, yarn properties

\section{Introduction}

It is desirable to design new functional yarns made from special materials in order to make the value of spun yarns higher. The materials for these yarns, however, are various and the spin-ability of these materials would be not high in general. In addition, small-scale productions would be demanded for these yarns [1]. Therefore, we.undertook to research on the new spinning system for new functional yarns that the principle of the spinning process differs from that in ordinary spinning systems in commercial use.

We have regard for the throstle-spinning machine, a kind of the twist draft spinning, since the machine has desirable properties for the development of the new spinning system as follows: simplicity of the pretreatment process; capability of extreme small lot and low spin-ability materials. Now we are developing the new spinning system based on the throstle-spinning machine, TCTDS (Tension Control type Twist Draft Spinning, Fig.1) that is improved on the yarn productivity using modern mechanical and electronic techniques [1,2]. In addition, an analysis of the fiber dynamics in the twist draft spinning including TCTDS is being carried out for making TCTDS more controllable. As a result of the analysis, the relation between diameter and twist rate of yarns spun with the twist draft spinning was revealed that is valuable tool in designing of yarns spun with TCTDS [3].

In the literature several other studies on the twist draft spinning are put forward: Liddy and Denby developed an experimental machine of the twist draft spinning and the coefficients of variation of mass per unit length of yarns produced by their machine were analyzed [4]. Matsumoto et al researched on the relation between mechanical factors of the throstle-spinning machine and the properties of the yarn

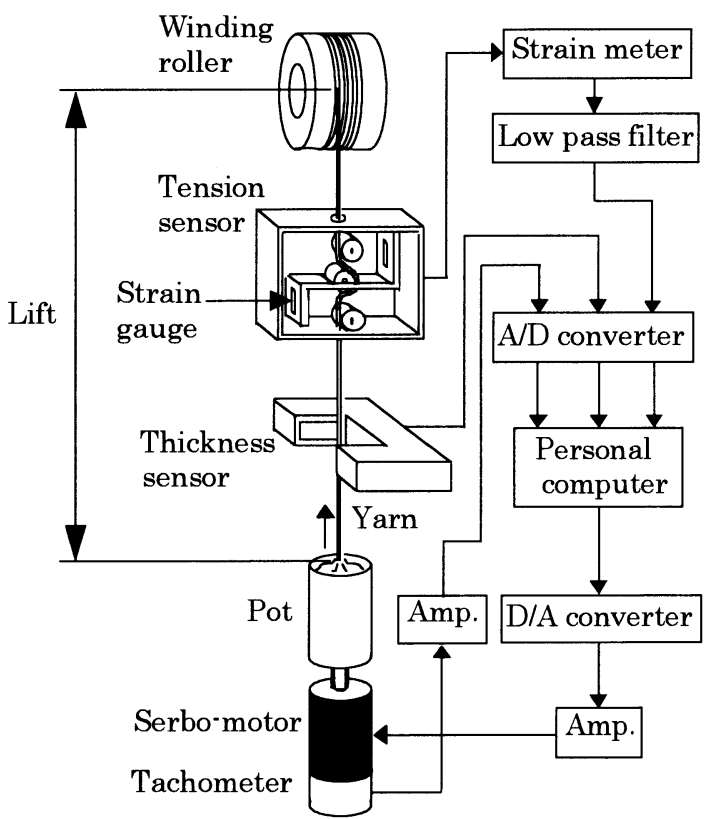

Fig.1 Schematic diagram of TCTDS

[5,6]. Matsumoto et al also produced the silk/raw-silk core-spun yarn by using an improved throstle-spinning machine [7]. But none of the reports have clarified both theoretically and experimentally the relation between the fiber properties and the properties of yarns spun with the twist draft spinning at all, besides our previous report [3].

This paper presents the further analysis of the fiber dynamics in the twist draft spinning and the derived result of the relation between linear density and twist rate of the twist draft spinning yarn. We will suggest an explanation for the relation between the theoretical data and the practical data of

* Corresponding author Fax: +81-268-37-1102, E-mail: takemura@pit-nagano.ac.jp 
yarn properties given by the related experiments.

\section{TCTDS}

Fig.1 shows a schematic diagram of TCTDS that is being developed by the authors. A pot is directly coupled to a servomotor with a tachometer. Rotational speed of the pot, i.e., twisting speed is measured with this tachometer and controlled by a computer. The measured value of twisting speed is saved in the computer. A tension sensor and a laser thickness sensor measure yarn tension and yarn thickness, respectively, and these data are stored in the computer. A winding roller is driven by a servomotor with a rotational speed controller.

The appearance of spinning in TCTDS is summarized as follows: a yarn is spun out directly from a treated large mass of fibers in the pot, twisted by the rotation of the pot, and wound up by the winding roller; Twisting and drafting of the yarn are performed simultaneously. A continuous rotation of the pot gives more twist to the yarn and leads yarn tension and thickness larger. At a stop of the rotation, the inverse phenomenon occurs. Taking this fact into account, the twisting control system that twist rate is operated by twisting speed so as to make yarn tension constant is designed in TCTDS for the purpose of keeping steady state condition of the yarn forming. The reason why near uniform yarns are attained by keeping yarn tension constant is that the number of fibers drawn out of the mass is approximately proportional to yarn tension on a condition that density of the mass is invariant.

The pretreatment process in TCTDS is simple: making a fleece by a card machine; rolling it in the direction perpendicular to fibers; packing it into the pot. On the contrary, that of ordinary spinning systems such as Ring spinning consists of extremely many processes. In addition, TCTDS needs material only about $0.02 \mathrm{~kg}$ for one lot as opposed to ordinary spinning systems that raw material for one lot is more than $1,000 \mathrm{~kg}$.

\section{Analysis of the fiber dynamics in the twist draft spinning}

Fig. 2 shows the yarn forming effect of the twist draft spinning. Some fibers of a top in the pot are entangled with a yarn being spun out and then these fibers are drawn out by the withdrawal force caused by winding of the yarn. Therefore, it is necessary for the steady yarn forming to maintain the holding of fibers by twist between the yarn and the top.

In our previous report, the dynamical condition for the maintenance of the holding was derived from an analysis of forces operating in this holding area, and the relation between diameter and twist rate of the twist draft spinning yarn was revealed by using the derived condition [3]. This result is reviewed at first of this section for an introduction of the problem. New results of the analysis concerning the relation between linear density and twist rate of the yarn will be shown at the rest.

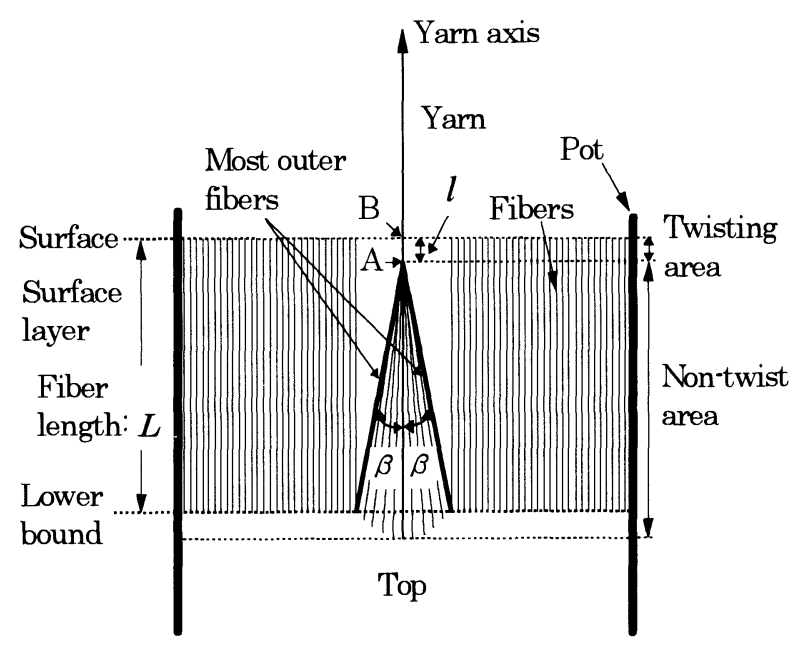

Fig.2 Yarn forming effect of the twist draft spinning

It is assumed for the analysis that the fibers are taken to be similar in all properties, i.e., length $L$, diameter $d$, and the coefficient of inter-fiber friction $\mu$, and the mass of fibers in the pot is regular.

\subsection{Geometrical condition of the holding of fibers by twist}

In Fig.2, point $\mathrm{A}$ is the lower end of the twisting area that twist of the yarn penetrates into the top and some fibers of the top are entangled with the yarn. The upper end of the twisting area is denoted by point $B$ that is the crossing of the yarn axis and the plane involving the surface of the top. The consecutive spread area of fibers held with the twisting area is denoted by the non-twist area. The layer, thickness of which is $L$ from the surface, is denoted by the surface layer.

Some assumptions are made as follows:

- The cross-sectional number of fibers $N$, twist rate $\lambda$ and diameter $D$ of the yarn including the twisting area are uniform. This is derived from steady state condition of the yarn forming.

- The angle $\beta$ between the yarn axis and the axes of fibers which are most outer from the yarn axis in the non-twist area is equal to the twist angle of fibers which lie on surface of the yarn. This is derived from the continuity of these areas.

- The geometry of the most outer fibers in the non-twist area is ideal, i.e., one end of each of the most outer fibers would be on $A$ and the other end would be on the lower bound of the surface layer.

On the assumptions, the length $l$ between $A$ to $\mathrm{B}$, i.e., the length of the twisting area is attained as follows [3]:

$$
l=L-L \cos \beta \cong \frac{L \pi^{2} \lambda^{2} D^{2}}{2}
$$

\subsection{Dynamical condition of the holding of fibers by twist}

Fibers in the twisting area and the non-twist area are clas- 
sified into four types as follows:

- The yarn side fiber, the upper end of which is over point $\mathrm{B}$;

- The entangled fiber, the upper end of which is between point $B$ and point $A$;

- The mass side fiber, the upper end of which is under point A;

- Both of the yarn side fiber and the entangled fiber is termed as the held fiber.

The dynamical condition of the holding can be expressed as follows by using the classification:

(Total of frictional forces acting between the entangled fibers and the yarn side fibers in the twisting area)

$=($ Total of frictional forces acting between the entangled fibers and the mass side fibers in the non-twist area)

Since sum of frictional forces acting between the held fibers and the mass side fibers is equal to yarn tension $F$, the right hand side of above condition can be represented as a part of $F$. Accordingly, the dynamical condition can be expressed as follows [8]:

$$
\mu N p I_{0}=r F
$$

where $p$ and $r$ are the average pressure on a fiber per unit length and the contributing ratio, respectively; $I_{0}$ is the effective length of contact [8] between the entangled fiber and the yarn side fiber in the twisting area.

\section{Analysis of the left hand side of Equation 2}

Fig.3 shows the cross-sectional distribution of fibers about the surface layer; where $x$ being measured along the yarn axis in the direction of yarn motion as point $\mathrm{A}$ being the origin. Functions $f(x), g(x)$ and $h(x)$ mean the number of the yarn side fibers, the entangled fibers and the held fibers, respectively, and are expressed as follows [8]:

$$
\begin{aligned}
& f(x)=\left\{\begin{array}{l}
\frac{N(x+L-l)}{L}(l-L \leq x \leq l) \\
0(-L \leq x<l-L)
\end{array}\right. \\
& g(x)=\left\{\begin{array}{c}
\frac{N(l-x)}{L}(0 \leq x \leq l) \\
\frac{N l}{L}(l-L \leq x<0) \\
\frac{N(x+L)}{L}(-L \leq x<l-L)
\end{array}\right. \\
& h(x)=\left\{\begin{array}{c}
N(0 \leq x \leq l) \\
\frac{N(x+L)}{L}(-L \leq x<0)
\end{array}\right.
\end{aligned}
$$

so that $I_{0}$ can be calculated as follows [8]:

$$
I_{0}=\int_{0}^{l} \frac{f(x)}{N} \frac{g(x)}{N} d x=\frac{(3 L-2 l) l^{2}}{6 L^{2}}
$$

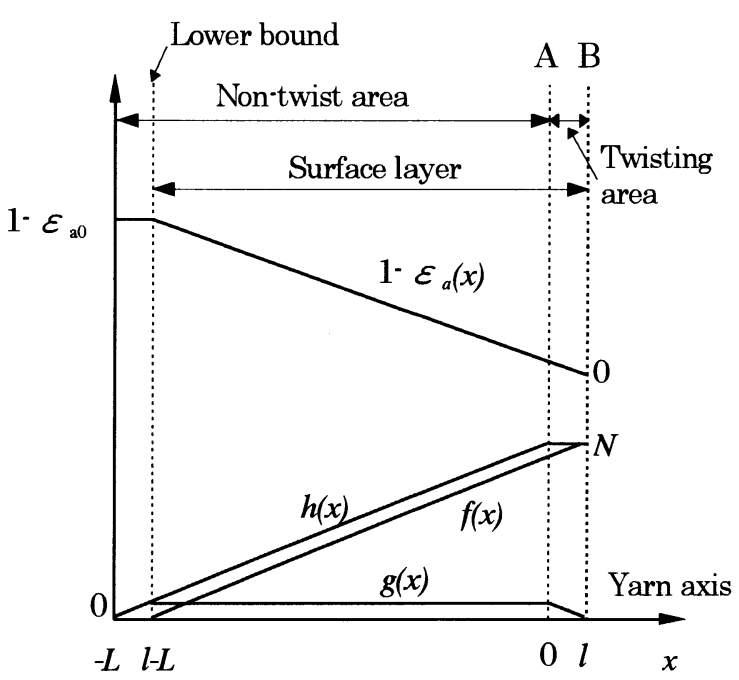

Fig.3 Cross-sectional distribution of fibers about the surface layer

In addition, $p$ can be attained as follows $[9,10]$ :

$$
\begin{aligned}
& p=\frac{2 d}{1-\varepsilon_{y}} P_{y} \\
& P_{y}=2 \pi \lambda^{2} F
\end{aligned}
$$

where $P_{y}$ and $\varepsilon_{y}$ are the average pressure and the average porosity ratio over the whole yarn, respectively. On the definition of the porosity ratio, the relation between $D$ and $N$ is given as follows:

$$
D^{2}=\frac{d^{2} N}{1-\varepsilon_{y}}
$$

Substituting Equations 1, 6, 7, 8 and 9 into the left hand side of Equation 2, the following function of $l$ is derived [3]:

$$
(\text { Left hand side of Equation } 2)=\frac{4 \mu(3 L-2 l) l^{3} F}{3 \pi d L^{3}}
$$

\section{Analysis of the right hand side of Equation 2}

Since the number of the held fibers is few enough to that of the mass side fibers, $F$ can be written as follows $[9,10]$ :

$$
F=\int_{-L}^{0} \mu h(x) \frac{2 d P}{1-\varepsilon} d x
$$

where $P_{t}$ and $\varepsilon_{t}$ are the pressure and the porosity ratio in the top, respectively. The relation between the pressure and the porosity ratio of fiber assemblages are approximately written as follows [10]:

$$
\begin{aligned}
& P_{y} \cong c_{y}\left(1-\varepsilon_{y}\right)^{3} \\
& P_{t} \cong c_{t}\left(1-\varepsilon_{t}\right)^{3}
\end{aligned}
$$


where $c_{y}$ and $c_{t}$ are constants. Substituting Equation 13 into Equation 11, $F$ can be rewritten as follows [3]:

$$
F=2 \mu d c_{t} \int_{-L}^{0} h(x)\left(1-\varepsilon_{t}\right)^{2} d x
$$

Similarly, the right hand side of Equation 2 is attained as follows [3]:

$$
r F=2 \mu d c_{t} \int_{-L}^{0} g(x)(1-\varepsilon)^{2} d x
$$

Because of both the pretreatment process and the regularity of the top, 1- $\varepsilon_{t}$ could be expressed as follows and be represented in Fig.3 [3]:

$$
1-\varepsilon_{t}=\left\{\begin{array}{c}
\left(1-\varepsilon_{t 0}\right) \frac{l-x}{L}(l-L \leq x \leq l) \\
1-\varepsilon_{t 0}(x<l-L)
\end{array}\right.
$$

where $\varepsilon_{t 0}$ is the porosity ratio of lower side of the top and constant. The ratio $r$ is attained by using Equation 14 to 16 , and shown as follows [3]:

$$
r \cong \frac{4 l}{L+4 l}
$$

\subsection{Relation between diameter and twist rate of the yarn in steady state condition of the yarn forming}

Substituting Equation 10 and 17 into Equation 2 and eliminating $F$, Equation 2 is expressed as follows by using newly defined non-dimensional variable $y=l / L[3]$ :

$$
y^{4}-\frac{5}{4} y^{3}-\frac{3}{8} y^{2}+\frac{3 \pi d}{8 \mu L}=0
$$

Let $y_{p}$ be the real root of Equation 18 lying in $(0,1)$. Substituting $y_{p}$ into Equation 1, the relation between $D$ and $\lambda$ is obtained as follows [3]:

$$
D \lambda=\frac{\sqrt{2 y_{p}}}{\pi}=C_{D \lambda}
$$

\subsection{Relation between linear density and twist rate of the yarn in steady state condition of the yarn forming}

Yarn tension $F$ is obtained as follows by calculating Equation 14:

$$
F=\frac{\mu d c_{t} N L\left(1-\varepsilon_{t 0}\right)^{2}\left(1+4 y_{p}\right)}{6}
$$

An assumption is made for the relation between the pressure and the porosity ratio of fiber assemblages expressed in Equations 12 and 13 as follows:

$$
c_{y}=c_{t}
$$

Substituting Equation 8, 12, 19, 20 and 21 into Equation 9 and eliminating $1-\varepsilon_{y}, P_{y}, D, F, c_{y}$ and $c_{t}$, the relation between $N$ and $\lambda$ is obtained as follows:

$$
\begin{aligned}
& \sqrt{N} \lambda=C_{D \lambda}^{\frac{3}{2}} d^{-\frac{5}{4}}\left(1-\varepsilon_{t 0}\right)^{\frac{1}{2}}\left(\frac{\pi \mu L\left(1+4 y_{p}\right)}{3}\right)^{\frac{1}{4}} \\
& =C_{N \lambda}
\end{aligned}
$$

Linear density of the yarn $W_{y}$ is expressed as follows:

$$
W_{y}=N W_{f}
$$

where $W_{f}$ is linear density of fiber. Equation 22 is rewritten as follows by using Equation 23:

$$
\sqrt{W_{y}} \lambda=\sqrt{W_{f}} C_{N \lambda}=K
$$

where $K$ is twist multiplier.

The finding of this section conveys that the relation described in Equation 24 is unique if the properties of raw material, i.e., fiber length, fiber diameter, the coefficient of inter-fiber friction and the porosity ratio in the top, are unique.

\section{Experimental}

Several yarns of each case shown in Table 1 were spun out at each range of set points of yarn tension shown in Table 2.

Mean twist rate of each yarn was measured in real time by TCTDS [2]. Mean linear density of each yarn was calculated from the mass determined when the test length was $2 \mathrm{~m}$.

The values of $\mu$ in Table 1 were measured by Röder's method[11]. The winding speed was set at $6.0 \times 10^{-3} \mathrm{~m} / \mathrm{s}$. The length of the lift was $0.6 \mathrm{~m}$. All experiments were performed in a room at temperature of $20^{\circ} \mathrm{C}$ and relative humidity of $60 \%$.

\section{Results and Discussion}

The relation between the square root of mean linear density $W_{y}^{1 / 2}$ and mean twist rate $\lambda$ of yarn of each case is shown in Fig.4 for case 1 and 2, Fig. 5 for case 3, and Fig.6 for case 4 and 5 . Both horizontal and vertical coordinates of Fig.4, 5 and 6 are graduated on a $\log$ scale.

Gradients of regression lines on Fig.4, 5 and 6 are shown in Table 3. It shows good agreement with the theoretical value of the gradient $(-1)$, represented in Equation 24.

Twist multiplier $K\left[\left(\right.\right.$ turns $\left.\left./ 10^{-3} \mathrm{~m}\right) \times\left(10^{-3} \mathrm{~kg} / \mathrm{m}\right)^{1 / 2}\right]$ for each case is calculated based on the data in Fig.4, 5 and 6, and shown in Table 4. In addition, the theoretical value of $K$ for each case is calculated based on Equation 24, and shown in Table 4, too.

Results of both Table 4 and Fig. 6 agree with Matumoto's result in respect of the throstle-spinning yarn [6] that $K$ becomes smaller when fiber length is longer.

As shown in Table 4, the theoretical value of $K$ is somewhat smaller than the experimental one for each case. Hannah mentioned the following factors that affect this matter [8]: 
Table 1 Properties of raw materials

\begin{tabular}{|l|l|l|l|l|l|}
\hline Case & 1 & 2 & 3 & 4 & 5 \\
\hline Fiber & $\mathrm{P}^{\mathrm{a}}$ & $\mathrm{P}^{\mathrm{a}}$ & $\mathrm{N}^{\mathrm{b}}$ & $\mathrm{P}^{\mathrm{a}}$ & $\mathrm{P}^{\mathrm{a}}$ \\
\hline$L\left[10^{-3} \mathrm{~m}\right]$ & 38 & 38 & 38 & 38 & 51 \\
\hline$d\left[10^{-6} \mathrm{~m}\right]$ & 12.4 & 12.4 & 19.0 & 17.4 & 17.4 \\
\hline$\mu$ & 0.35 & 0.35 & 0.30 & 0.37 & 0.37 \\
\hline $1-\varepsilon_{t 0}$ & 0.10 & 0.072 & 0.15 & 0.10 & 0.10 \\
\hline$W_{y}\left[10^{-6} \mathrm{~kg} / \mathrm{m}\right]$ & 0.17 & 0.17 & 0.33 & 0.33 & 0.33 \\
\hline
\end{tabular}

${ }^{\mathrm{a}}$ Polyester, ${ }^{\mathrm{b}}$ Nylon 6

Table 2 Ranges of set points of yarn tension for spinning

\begin{tabular}{|l|l|l|l|l|l|}
\hline Case & 1 & 2 & 3 & 4 & 5 \\
\hline Yarn & 0.14 & 0.14 & 0.19 & 0.19 & 0.24 \\
tension & $\sim$ & $\sim$ & $\sim$ & $\sim$ & $\sim$ \\
{$[N]$} & 0.49 & 0.39 & 0.44 & 0.44 & 0.49 \\
\hline
\end{tabular}

Table 3 Gradients of regression lines on Fig.4, 5 and 6

\begin{tabular}{|l|l|l|l|l|l|}
\hline Case & 1 & 2 & 3 & 4 & 5 \\
\hline Gradient & -1.0 & -0.94 & -1.0 & -0.91 & -0.98 \\
\hline
\end{tabular}

Table 4 Twist multiplier $K$ [ (turns $\left.\left./ 10^{-3} \mathrm{~m}\right) \times\left(10^{-3} \mathrm{~kg} / \mathrm{m}\right)^{1 / 2}\right]$

\begin{tabular}{|l|l|l|l|l|l|}
\hline Case & 1 & 2 & 3 & 4 & 5 \\
\hline Exper. & 0.087 & 0.079 & 0.10 & 0.093 & 0.091 \\
\hline Theoret. & 0.064 & 0.054 & 0.076 & 0.067 & 0.064 \\
\hline
\end{tabular}

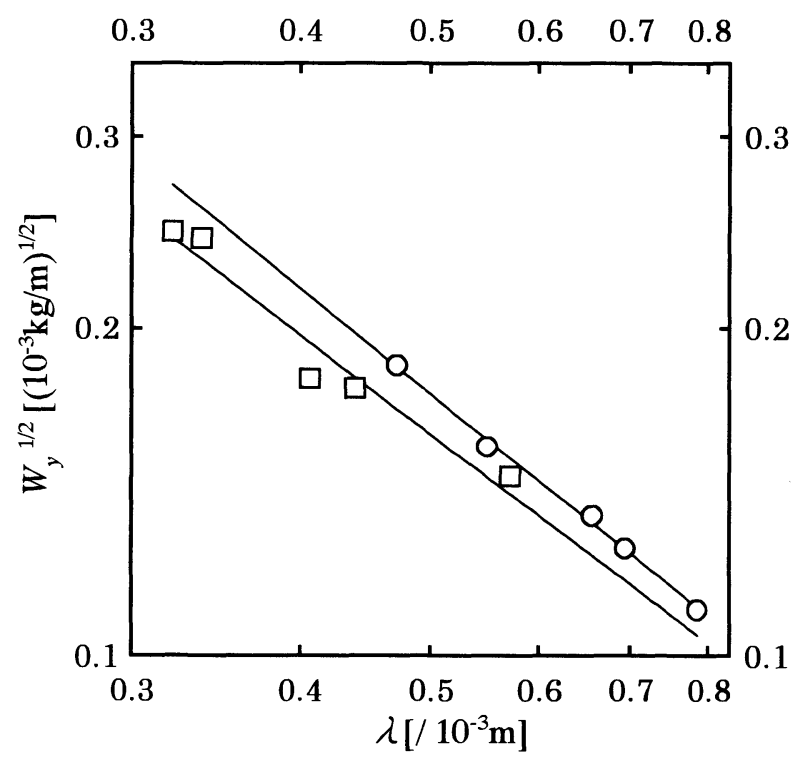

Fig.4 Relation between the square root of mean linear density $W_{y}^{1 / 2}$ and mean twist rate $\lambda$ of yarns of case 1 and 2 . Symbols of $\bigcirc$ and $\square$ are corresponding to case 1 and 2 , respectively.

- In a real yarn, the effective total surface area over which fibers are in contact is noticeably less than the total surface area of fibers, since the peripheral fibers are then a substantial proportion of the whole;

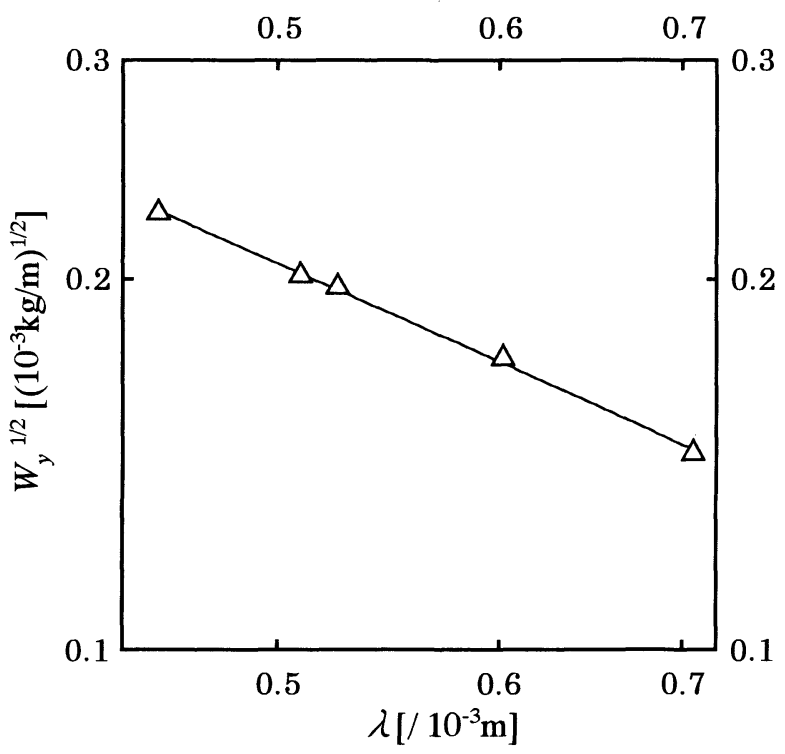

Fig.5 Relation between the square root of mean linear density $W_{y}^{1 / 2}$ and mean twist rate $\lambda$ of yarns of case 3 .

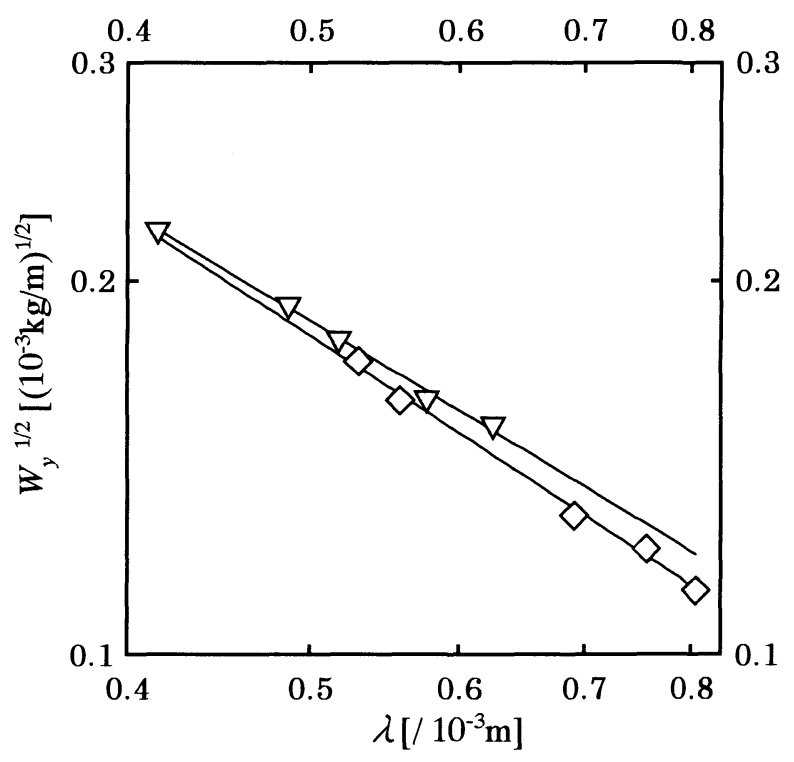

Fig. 6 Relation between the square root of mean linear density $W_{y}^{1 / 2}$ and mean twist rate $\lambda$ of yarns of case 4 and 5. Symbols of $\nabla$ and $\diamond$ are corresponding to case 4 and 5 , respectively.

- Since the total area of a cross-section of a real yarn is $\left(1-\varepsilon_{t}\right)^{-1}$ times greater than for a ideal yarn with close packing of fibers, the contact length of fibers in a real yarn may be taken to be reduced in the ratio $\left(1-\varepsilon_{t}\right)^{1 / 2}$.

Equation 24 is considered, however, to be adequate to express the relation between linear density and twist rate of yarn because of the reasons as follows:

- The changing aspect of the theoretical value of $K$ agrees with the experimental one at various properties of raw materials;

- Independently of the cases, the experimental value and the theoretical value of $K$ is approximately in the ratio of $10: 7$. 


\section{Conclusions}

Through this paper, an analysis of the fiber dynamics in the twist draft spinning is performed. As a result, the relation between linear density and twist rate of the twist draft spinning yarn is revealed both theoretically and experimentally.

The finding of this paper is useful for designing of yarns of the twist draft spinning. For example, in case of raw materials of synthetic fibers, it is possible to obtain sets of yarns of different properties by changing cut length and denier of filaments, and density of the mass of fibers in the pot. Of course, the yarn properties are predictable by Equation 24 and time-consuming trials for designing may be circumvented in this way.

\section{References}

[1] Nakazawa. M. et al; J. Text. Mach. Soc. Japan, 42, 72 (1996).

[2] Huang, G. et al; Sen'i Gakkaishi, 54, 32(1998).

[3] Takamura. H. et al; Text. Res. J., (in press).

[4] Liddy, D. T., Denby, E. F.; J. Text. Inst., 51, T1109 (1960).

[5] Matsumoto, Y. et al; J. Text. Mach. Soc. Japan, 46, T215 (1993)

[6] Matsumoto, Y. et al; J. Text. Mach. Soc. Japan, 47, T83 (1994)

[7] Matsumoto, Y. et al; J. Text. Mach. Soc. Japan, 47, T219 (1994)

[8] Hannah, M.; J. Text. Inst., 41, T57 (1950).

[9] Cox, D. R.; J. Text. Inst., 43, T87 (1952).

[10]Smith, P. A.; J. Text. Inst., 53, T511 (1962).

[11]Röder, H. L.; J. Text. Inst., 44, T247 (1953). 